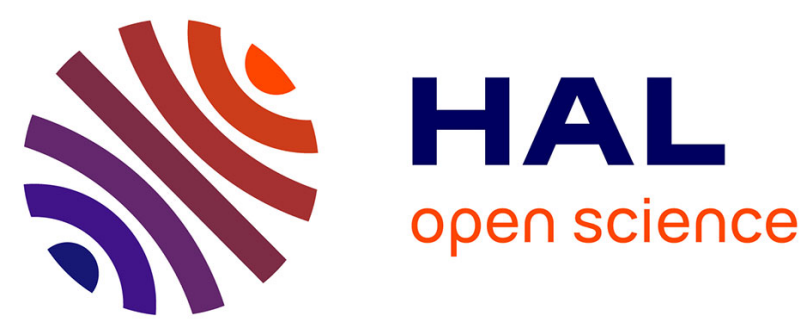

\title{
Coronal mass ejections and solar wind mass fluxes over the heliosphere during solar cycles 23 and 24 (1996-2014)
}

Philippe Lamy, Olivier Floyd, Eric Quémerais, Bice Boclet, Stéphane Ferron

\section{To cite this version:}

Philippe Lamy, Olivier Floyd, Eric Quémerais, Bice Boclet, Stéphane Ferron. Coronal mass ejections and solar wind mass fluxes over the heliosphere during solar cycles 23 and 24 (1996-2014). Journal of Geophysical Research Space Physics, 2017, 122 (1), pp.50-62. 10.1002/2016JA022970 . insu01405119

\section{HAL Id: insu-01405119 \\ https://hal-insu.archives-ouvertes.fr/insu-01405119}

Submitted on 7 Sep 2020

HAL is a multi-disciplinary open access archive for the deposit and dissemination of scientific research documents, whether they are published or not. The documents may come from teaching and research institutions in France or abroad, or from public or private research centers.
L'archive ouverte pluridisciplinaire HAL, est destinée au dépôt et à la diffusion de documents scientifiques de niveau recherche, publiés ou non, émanant des établissements d'enseignement et de recherche français ou étrangers, des laboratoires publics ou privés. 


\section{Journal of Geophysical Research: Space Physics}

\section{RESEARCH ARTICLE \\ 10.1002/2016JA022970 \\ Key Points: \\ Coronal mass ejections and solar wind mass fluxes over the heliosphere during solar cycles 23 and 24 (1996-2014)}

- Assess the contribution of coronal mass ejections to solar wind mass flux usingSOHO/LASCO, SOHO/SWAN, and OMNI data

- Their ratio closely tracked the solarcycle over the heliosphere - It varied from negligible during the solar minima of cycles $22 / 23$ and $23 / 24$ to 6 and $5 \%$ at the maximum of cycles 23 and 24 , respectively

Correspondence to: P. Lamy,

philippe.lamy@lam.fr

\section{Citation:}

Lamy, P., O. Floyd, E. Quémerais, B. Boclet, and S. Ferron (2017), Coronal mass ejections and solar wind mass fluxes over the heliosphere during solar cycles 23 and 24 (1996-2014), J. Geophys. Res., 122, 50-62, doi:10.1002/2016JA022970.

Received 19 MAY 2016 Accepted 16 NOV 2016 Accepted article online 22 NOV 2016 Published online 19 JAN 2017

○2016. American Geophysical Union. All Rights Reserved.

\author{
P. Lamy ${ }^{1,2}$, O. Floyd ${ }^{3}$ (D) E. Quémerais ${ }^{2}$, B. Boclet ${ }^{1}$, and S. Ferron ${ }^{4}$ \\ ${ }^{1}$ Laboratoire d'Astrophysique de Marseille, CNRS \& Aix-Marseille Université, Marseille, France, ${ }^{2}$ Laboratoire Atmosphères, \\ Milieux et Observations Spatiales, CNRS \& Université de Versailles Saint-Quentin-en-Yvelines, Guyancourt, France, ${ }^{3}$ AKKA \\ Informatique et Systèmes, Toulouse, France, ${ }^{4}$ ACRI-ST, Sophia Antipolis, France
}

\begin{abstract}
Coronal mass ejections (CMEs) play a major role in the heliosphere, and their contribution to the solar wind mass flux, already considered in the Skylab and Solwind eras with conflicting results, is reexamined in the light of 19 years (1996-2014) of SOHO observations with the Large Angle and Spectroscopic Coronagraph (LASCO-C2) for the CMEs and extended for the first time to all latitudes thanks to the whole-heliosphere data from the Solar Wind ANisotropies (SWAN) instrument supplemented by in situ data aggregated in the OMNI database. First, several mass estimates reported in the ARTEMIS (Automated Recognition of Transient Events and Marseille Inventory from Synoptic maps) catalog of LASCO CMEs are compared with determinations based on the combined observations with the twin STEREO/Sun Earth Connection Coronal and Heliospheric Investigation coronagraphs in order to ascertain their validity. A simple geometric model of the CMEs is introduced to generate Carrington maps of their mass flux and then to produce annualized synoptic maps. The Lyman $\alpha$ SWAN data are inverted to similarly produce synoptic maps to be compared with those of the CME flux. The ratio of the annualized CME to solar wind mass flux is found to closely track the solar cycle over the heliosphere. In the near-ecliptic region and at latitudes up to $\sim 55^{\circ}$, this ratio was negligibly small during the solar minima of cycles $22 / 23$ and $23 / 24$ and rose to $6 \%$ and $5 \%$, respectively, at the maximum of solar cycles 23 and 24 . These maximum ratios increased at higher latitudes, but this result is likely biased by the inherent limitation of determining the true latitude of CMEs.
\end{abstract}

\section{Introduction}

It is now well established that coronal mass ejections (CMEs) play a major role in the heliosphere, starting from the corona to interplanetary space and including their interaction with planets. The disturbances that they create largely control the space weather activity, injecting significant amounts of mass and energy in large volumes of interplanetary space which further vary approximately in phase with the solar cycle. The question of their quantitative contribution to the background solar wind (SW) mass flux is important to take stock of the global balance in interplanetary space and was addressed as soon as sufficient data became available. Hildner [1977] used Skylab coronagraph images taken from May 1973 to February 1974 ( $\approx 2$ years before the solar minimum of cycle 20/21) and estimated that they probably contributed at least $3 \%$ of the mass flux of the solar wind. He then predicted a value of more than $10 \%$ at the next solar cycle maximum on the assumption that the frequency of CMEs would increase correspondingly. These values were later revised to respectively $10 \%$ and $16 \%$ by Webb and Howard [1994] on the grounds that Hildner did not correct for the latitude variation of the CMEs. Howard et al. [1985] used Solwind coronagraph images taken from 1979 to 1981 at the maximum of solar cycle 21 and estimated a CME contribution of only 5\%. Webb and Howard [1994] revised this previous analysis, introducing several corrections and extending the time interval to 1984. Further combining with Skylab and SMM (Solar Maximum Mission) data, they were able to estimate the mass flux of CMEs over more than a complete 11 year solar cycle (prominently solar cycle 21) from 1973 to 1985. Then using published solar wind data from the IMP spacecraft and Helios, they found that the ratio of the annualized CME to solar wind mass flux tends to track the solar cycle and that near its maximum, CMEs could provide $\approx 15 \%$ of the average mass flux to the near-ecliptic solar wind. Jackson and Howard [1993] estimated that the global Solwind mass during 1979 to 1981 was $\approx 16 \%$ of the Helios solar wind mass based on an exponential integration of the Solwind mass curve. 
The purpose of the present article is to reexamine this question for a different and longer period of solar activity, from 1996 to 2014 inclusive, thus covering solar cycle 23 and the rising phase of cycle 24, and to rely on the homogeneous CME data set from a single instrument, the Large Angle and Spectroscopic Coronagraph (LASCO-C2) [Brueckner et al., 1995] on board the SOHO spacecraft, thus avoiding intercalibration problems. The analysis considers not only the near-ecliptic region for which several spacecraft provide solar wind data but also the whole heliosphere thanks to solar wind data collected by the Solar Wind ANisotropies (SWAN) instrument [Bertaux et al., 1995] also on board SOHO. It is organized as follows. Section 2 introduces the CME data set and takes advantage of recent determinations of CME mass to validate this data set. Section 3 explains how we calculate the heliospheric distribution of the CME mass flux for each Carrington rotation, discusses inherent limitations, and compares the annualized mass flux of the CMEs to that of the solar wind in the ecliptic recorded by in situ spacecraft. Section 4 introduces the SWAN solar wind measurements, explains how we calculate the heliospheric distribution of its mass flux, and compares the CME and SW heliospheric distributions of mass flux. We finally summarize our main results in section 5 .

\section{Coronal Mass Ejections}

\subsection{Coronal Mass Ejections Data Set}

To construct the heliospheric distribution of the mass flux of CMEs, we rely on the ARTEMIS-II catalog [Floyd et al., 2013]. Unlike other catalogs which use the LASCO images themselves, their automated detection and characterization is performed on synoptic maps built from time series of LASCO-C2 images first corrected for instrumental effects, calibrated in units of mean solar radiance, and resized to a common format of $512 \times 512$ pixels. These images are transformed to polar coordinates with two different angular steps: $0.4^{\circ}$ for radial distances of 3 and $3.5 R_{\odot}$ and $0.25^{\circ}$ beyond. A uniform linear resampling with a time step of 27.3 days $/ 1440=27.3 \mathrm{~min}$ is applied in order to remove the effect of irregular image acquisition. These maps have therefore a format of $1440 \times 900$ pixels at radial distance of 3 and $3.5 R_{\odot}$ and of $1440 \times 1440$ pixels at larger distances. This temporal resolution of $27.3 \mathrm{~min}$ is comparable to the average temporal cadence ( $\approx 24 \mathrm{~min}$ ) of the C2 images during 12 years (1999-2010), corresponding to $\approx 60$ images per day. In late 2010 , the cadence was increased to $\approx 105$ images per day, but the temporal resolution of the synoptic maps was maintained to $27.3 \mathrm{~min}$ to avoid introducing a bias in the detection rate of CMEs. The detection method has been described in detail by Boursier et al. [2009b] and updated by Floyd et al. [2013]. The ARTEMIS-II catalog lists the CMEs detected since March 1996 along with several of their attributes such as time of detection, central apparent latitude, angular width, and for a large fraction of them $(\approx 60 \%)$, mass and kinetic energy. For the time interval considered in this study, from 1996 to 2014 inclusive, the ARTEMIS-II catalog lists a total of 33,224 CMEs, of which 20,505 have their mass determined. As a matter of comparison, the Coordinated Data Analysis Workshop Data Center (http://cdaw.gsfc.nasa.gov/CME_list/) catalog lists a total of 24,854 CMEs, of which 16,478 have their mass determined.

The calculation of the mass of each CME from the Thomson scattering by its electron content requires several strong assumptions, in particular, that it lies close to the plane of the sky ("limb event"), since we have no longitudinal information from the images. In order to validate their determinations, Floyd et al. [2013] compared their results for seven CMEs with those independently obtained by Colaninno and Vourlidas [2009] that exploited the two independent views offered by the Sun Earth Connection Coronal and Heliospheric Investigation (SECCHI) coronagraphs of the STEREO mission. The direction of propagation is then determined by imposing the constraint that the same mass should be derived from the two stereoscopic views, thus leading to a more robust result. The agreement was generally excellent except for two cases for which the discrepancy could be understood. There is obviously a need to broaden the comparison as CME mass is a key parameter in our procedure to calculate mass flux. We thus took advantage of the recent work of Bein et al. [2013] which extends that of Colaninno and Vourlidas [2009] to 25 CMEs later observed with the SECCHI coronagraphs as presented below.

\subsection{Mass Estimation of Coronal Mass Ejections From LASCO and SECCHI}

The SECCHI observations reported by Bein et al. [2013] were performed from December 2007 to April 2011, with the bulk of them after December 2009, when both STEREO spacecraft were at more than $60^{\circ}$ from the Sun-Earth line, implying significantly different viewing geometries compared to SOHO. Actually, the relevant data are not given in the article of Bein et al. [2013] but were made available to us by one of its coauthor M. Temmer for each of the 25 CMEs: date and time of appearance as seen by the STEREO Extreme Ultraviolet 
Table 1. Comparison of the Mass of 21 CMEs Listed in the ARTEMIS-II Catalog and in Bein et al.[2013] ${ }^{\text {a }}$

\begin{tabular}{|c|c|c|c|c|c|c|c|}
\hline \multirow[b]{2}{*}{ CME Number } & \multirow[b]{2}{*}{ Date } & \multirow[b]{2}{*}{ Angle $\left({ }^{\circ}\right)$} & \multicolumn{3}{|c|}{ Bein et al. [2013] } & \multicolumn{2}{|c|}{ ARTEMIS-II } \\
\hline & & & Time & $\mathrm{m}_{0}(\mathrm{~g})$ & $\mathrm{m}_{14}(\mathrm{~g})$ & Time & $\mathrm{m}_{A}(\mathrm{~g})$ \\
\hline 1 & 31-Dec-2007 & 3 & $00: 43$ & $3.8 \mathrm{E} 15$ & $9.0 \mathrm{E} 15$ & $02: 27$ & $8.4 \mathrm{E} 15$ \\
\hline 2 & 2-Jan-2008 & 28 & $07: 28$ & 3.3E15 & 7.1E15 & $11: 58$ & $5.0 \mathrm{E} 15$ \\
\hline 3 & 26-Apr-2008 & 84 & $14: 26$ & 4.2E15 & $6.9 \mathrm{E} 15$ & $16: 03$ & $1.0 \mathrm{E} 15$ \\
\hline 4 & 16-Dec-2009 & 69 & $00: 46$ & $1.4 \mathrm{E} 15$ & 2.7E15 & $02: 42$ & $1.1 \mathrm{E} 15$ \\
\hline 5 & 10-Feb-2010 & 51 & $16: 00$ & $9.5 \mathrm{E} 14$ & $1.5 \mathrm{E} 15$ & $19: 00$ & $7.8 \mathrm{E} 14$ \\
\hline 6 & 3-Apr-2010 & 69 & 08:40 & $1.8 \mathrm{E} 15$ & 4.0E15 & $11: 36$ & $1.3 \mathrm{E} 15$ \\
\hline 7 & 7-Aug-2010 & 64 & 18:01 & 2.9E15 & $8.2 \mathrm{E} 15$ & $19: 21$ & $5.2 \mathrm{E} 15$ \\
\hline 8 & 14-Aug-2010 & 78 & $09: 26$ & 4.2E15 & 4.5E15 & $11: 30$ & $7.3 \mathrm{E} 15$ \\
\hline 9 & 27-Aug-2010 & 57 & $10: 10$ & $2.3 \mathrm{E} 15$ & 4.2E14 & $12: 04$ & $3.0 \mathrm{E} 15$ \\
\hline 10 & 31-Aug-2010 & 44 & $20: 40$ & $1.3 \mathrm{E} 15$ & 4.9E15 & $22: 04$ & $3.2 \mathrm{E} 15$ \\
\hline 11 & 2-Sep-2010 & 45 & 09:00 & $1.5 \mathrm{E} 15$ & $1.5 \mathrm{E} 15$ & $11: 20$ & 7.4E14 \\
\hline 12 & 30-Nov-2010 & 43 & $18: 25$ & $2.0 \mathrm{E} 15$ & $2.8 \mathrm{E} 15$ & $20: 35$ & $1.2 \mathrm{E} 15$ \\
\hline 13 & 14-Dec-2010 & 54 & $14: 53$ & 3.9E15 & $6.6 \mathrm{E} 15$ & $16: 40$ & $5.2 \mathrm{E} 15$ \\
\hline 14 & 18-Jan-2011 & 73 & 03:10 & $1.6 \mathrm{E} 15$ & 2.4E15 & 05:29 & $3.6 \mathrm{E} 15$ \\
\hline 15 & 31-Jan-2011 & 85 & $16: 15$ & 2.4E15 & 2.6E15 & $18: 27$ & $5.0 \mathrm{E} 15$ \\
\hline 16 & 1-Feb-2011 & 85 & $22: 16$ & $1.0 \mathrm{E} 15$ & 4.5E15 & $00: 52^{b}$ & 4.3E14 \\
\hline 17 & 13-Feb-2011 & 80 & $17: 10$ & 2.2E15 & $3.6 \mathrm{E} 15$ & $21: 17$ & 2.7E14 \\
\hline 18 & 15-Feb-2011 & 87 & 01:06 & $2.3 \mathrm{E} 15$ & $5.8 \mathrm{E} 15$ & 03:39 & 4.4E15 \\
\hline 19 & 7-Mar-2011 & 80 & $13: 48$ & 2.1E15 & 4.3E15 & $15: 57$ & $3.3 \mathrm{E} 15$ \\
\hline 20 & 21-Mar-2011 & 28 & 02:00 & 8.9E15 & 4.0E16 & 03:27 & $1.5 \mathrm{E} 16$ \\
\hline 21 & 7-Apr-2011 & 65 & $11: 18$ & 4.6E15 & $9.3 \mathrm{E} 15$ & $13: 07$ & 8.7E15 \\
\hline
\end{tabular}

Imagers (EUVI), $\mathrm{m}_{0}$ a deprojected mass derived from a fit to the COR 1 and COR2 data (correcting from the parts of the CME hidden by the occulting disk), $\mathrm{m}_{14}$ a deprojected mass calculated at a heliocentric distance of $14 \mathrm{R}_{\odot}$ from the COR2 data, and the angle between the trajectory of the CME and the plane of the sky perpendicular to the Sun-Earth direction (i.e., the LASCO sky plane).

The association of the CMEs detected by the LASCO-C2 and EUVI instruments was performed using the dates and times of appearance allowing for a difference of at most a few hours. Out of the 25 listed CMEs, 21 events with their mass reported in the ARTEMIS-II catalog were unambiguously associated. Following a

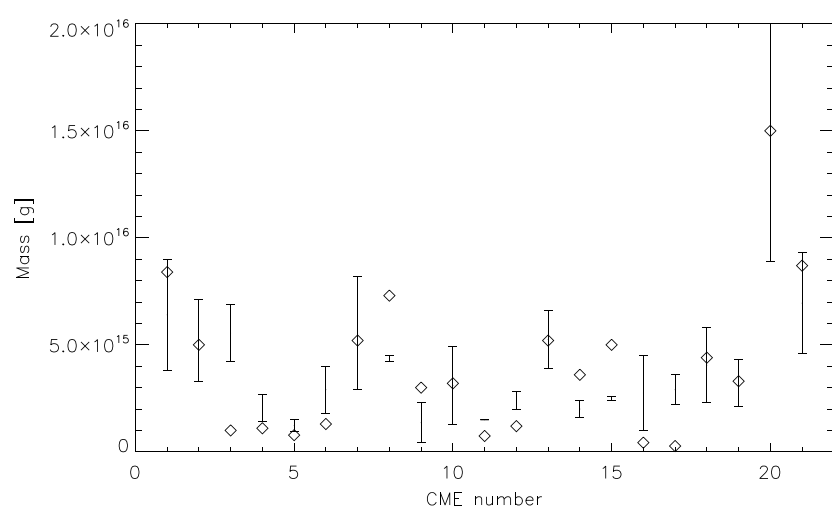

Figure 1. Comparison of the mass of 21 CMEs listed in the ARTEMIS-II catalog and in Bein et al. [2013]. The diamonds correspond to the ARTEMIS-II data and the vertical bars to the ranges delimited by the $\mathrm{m}_{0}$ and $\mathrm{m}_{14}$ values (see text for detail). recommendation from $M$. Temmer, we compared the ARTEMIS mass with both $\mathrm{m}_{0}$ and $\mathrm{m}_{14}$ as they should bracket the former mass. Indeed, $\mathrm{m}_{0}$ is estimated from the COR1 coronagraphs below $3 \mathrm{R}_{\odot}$, whereas $\mathrm{m}_{14}$ is intended to reflect the possible accumulation of solar wind material in front of the CMEs ("snow plow") as they travel outward. Table 1 lists the CME parameters and masses $\mathrm{m}_{0}, \mathrm{~m}_{14}$, and $\mathrm{m}_{A}$ (for ARTEMIS). Figure 1 displays these masses and indicates a surprisingly good general agreement between the two different methods. For 10 CMEs, the ARTEMIS mass $m_{A}$ lies between $\mathrm{m}_{0}$ and $\mathrm{m}_{14} ; \mathrm{m}_{A}$ exceeds $\mathrm{m}_{14}$ for four CMEs, whereas it is below $m_{0}$ for 

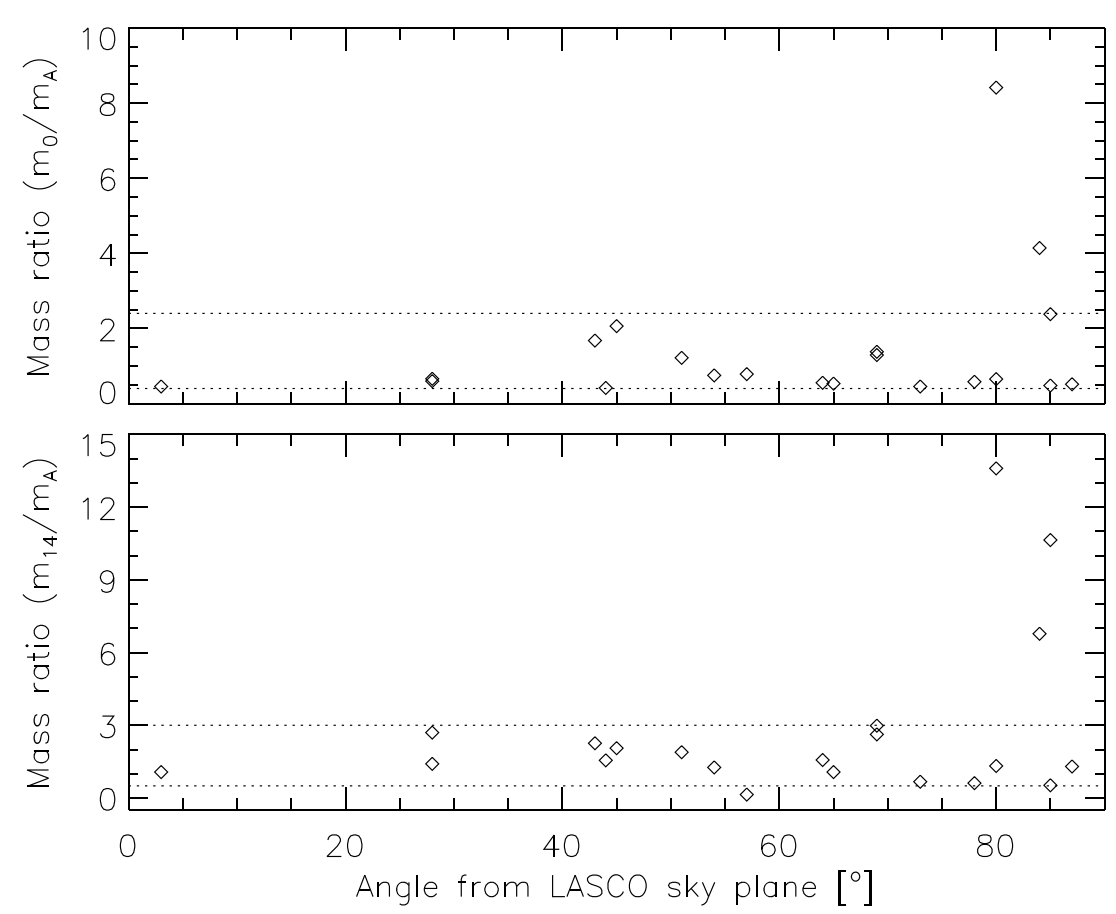

Figure 2. Ratio of the mass of CMEs listed in Bein et al. [2013] to those given in the ARTEMIS-II catalog as a function of their angle from the LASCO plane of the sky. (top) The $m_{0}$ and (bottom) the $m_{14}$ values (see text for detail).

seven CMEs. This constitutes indeed a highly satisfactory achievement granting that the masses were derived from different instruments and with different methods. Figure 2 displays the ratios $\mathrm{m}_{0} / \mathrm{m}_{A}$ and $\mathrm{m}_{14} / \mathrm{m}_{A}$ as a function of the angle from the plane of the sky. The $m_{0} / m_{A}$ ratios lie in the range 0.5 to 2.3 (marked with horizontal dotted lines) in all but two cases yielding an average value of 0.9 . The $\mathrm{m}_{14} / \mathrm{m}_{A}$ ratios lie in the range 0.5 to 3 in all but three cases. We emphasize that all these "outliers" are at angles larger $\geq 80^{\circ}$ (halo CMEs as seen from LASCO) where the projection effect (leading to underestimating the ARTEMIS mass) is expected to be the most pronounced. We conclude that, in general, ARTEMIS gives a good estimate of the initial mass of most CMEs.

\section{Coronal Mass Ejections Mass Flux}

\subsection{Calculation of the CME Mass Flux}

The heliospheric distribution of the mass flux is first calculated for each Carrington rotation (CR) on a grid of $90 \times 90$ pixels, that is with steps of $4^{\circ}$ in longitude and $2^{\circ}$ in latitude, ample for a later comparison with the SWAN data.

We need to define a three-dimensional geometry of the CME and implement the simple axisymmetric spherical cap model [Boursier et al., 2009a] whose subtended cone angle is equal to the angular width of the CME, and with its axis defined by the radial direction from the center of the Sun to the center of the CME. The mass of a CME is assumed to be uniformly distributed over the surface of the spherical cap. Whereas the apparent latitude of the axis is straightforwardly defined by the midpoint of the angular extent of the CME, its longitude basically remains unconstrained. We consider that all CMEs with an angular width less than $180^{\circ}$ have their axis in the instantaneous plane of the sky at the time of their detection, at the corresponding east or west limbs. CMEs whose width exceeds $180^{\circ}$ (1.5\% of the total population) are assumed to propagate along the instantaneous Sun-Earth line, either toward the Earth or away from it. Accordingly, they are divided into two equal mass sub-CMEs propagating in the two opposite directions. We then calculate the contribution of a CME in each pixel of the relevant Carrington map, sum up the contributions from all CMEs detected during the Carrington rotation, and divide by its duration to obtain the mass flux in units of $\mathrm{g} \mathrm{cm}^{-2} \mathrm{~s}^{-1}$. The CMEs are assumed to expand self-similarly so the flux may be calculated at any heliocentric distance. As a matter of convenience, we consider a distance of 1 AU since the SWAN and OMNI solar wind data to be used later for comparison are available at that distance. In the above calculation of the mass flux, CMEs are assumed to be 

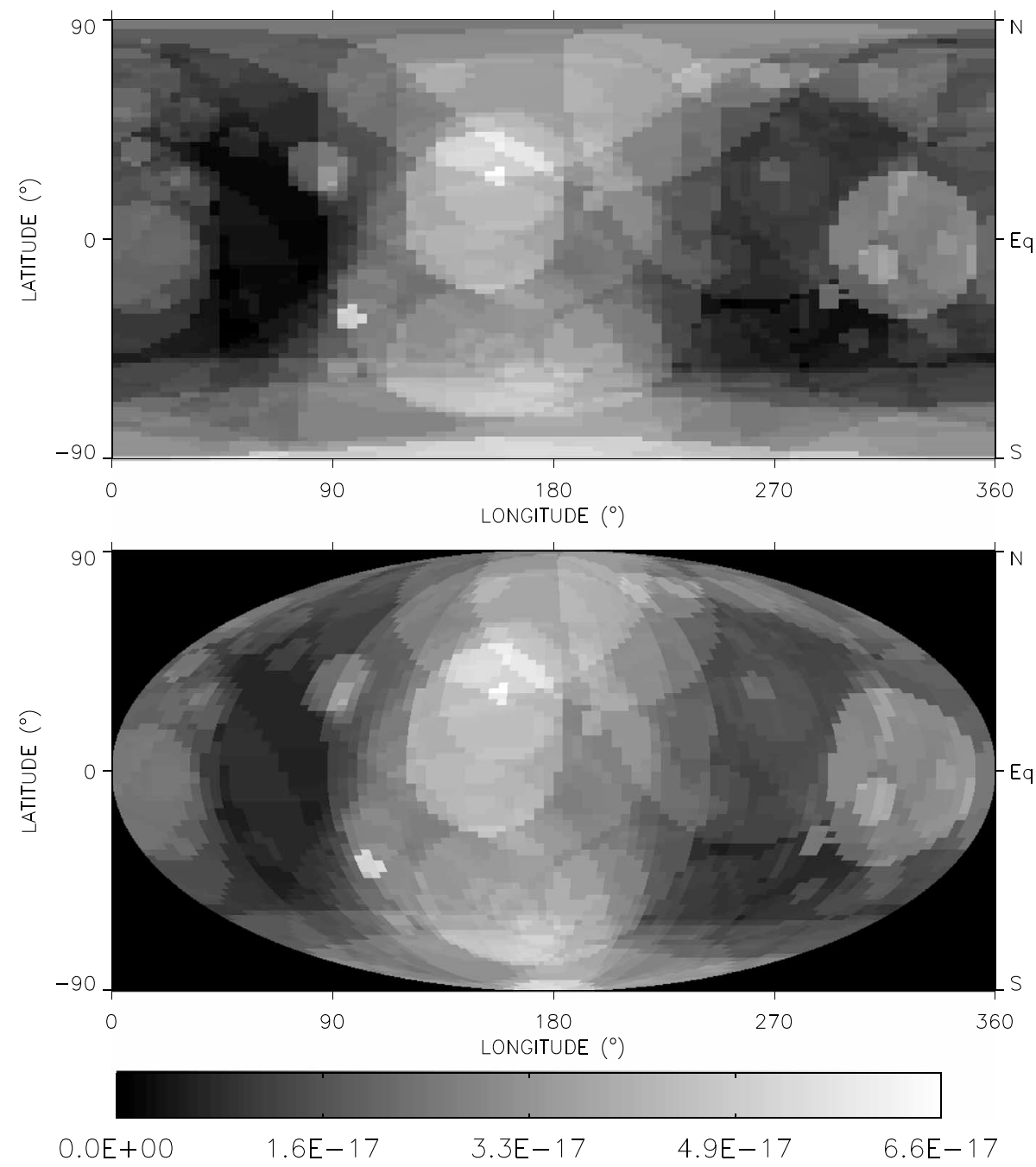

Figure 3. Two maps of the proton mass flux of CMEs corresponding to Carrington rotation 1970. (top) The display of the standard cylindrical projection and (bottom) the Mollweide projection.

composed of $90 \%$ protons and $10 \% \alpha$ particles. As the SWAN and OMNI data concern only protons, we scale the above CME mass flux to its proton component for obvious consistency reasons. Consequently, our results are reported in either proton number flux in units of proton $\mathrm{cm}^{-2} \mathrm{~s}^{-1}$ (in short $\mathrm{cm}^{-2} \mathrm{~s}^{-1}$ ) or proton mass flux in units of $\mathrm{g} \mathrm{cm}^{-2} \mathrm{~s}^{-1}$.

Figure 3 illustrates the case of CR 1970 in both standard cylindrical (top) and Mollweide (bottom) projections. The former presents the disadvantage of distorting the polar regions, whereas the latter one being equal area gives a correct rendering of the spatial distribution of the flux. The Carrington maps are combined to produce yearly maps which represent the annualized CME mass flux. Figure 4 displays six examples for years 1997, $2000,2003,2006,2009$, and 2013 spanning the whole time interval considered in the present study. Note the different brightness scales required to cope with the large dynamics resulting from the variation of the flux with solar activity. We finally construct a multiyear synoptic map over the whole time interval to which 20,505 CMEs contribute and which will be later presented when it will be compared with the similar map of the solar wind flux.

\subsection{Limitations}

As stated above, we had to make several strong assumptions to calculate the CME mass flux which we now discuss and show that they are not unduly restrictive. Indeed, they are mitigated by the fact that we are interested in long-term, typically yearly variations. 

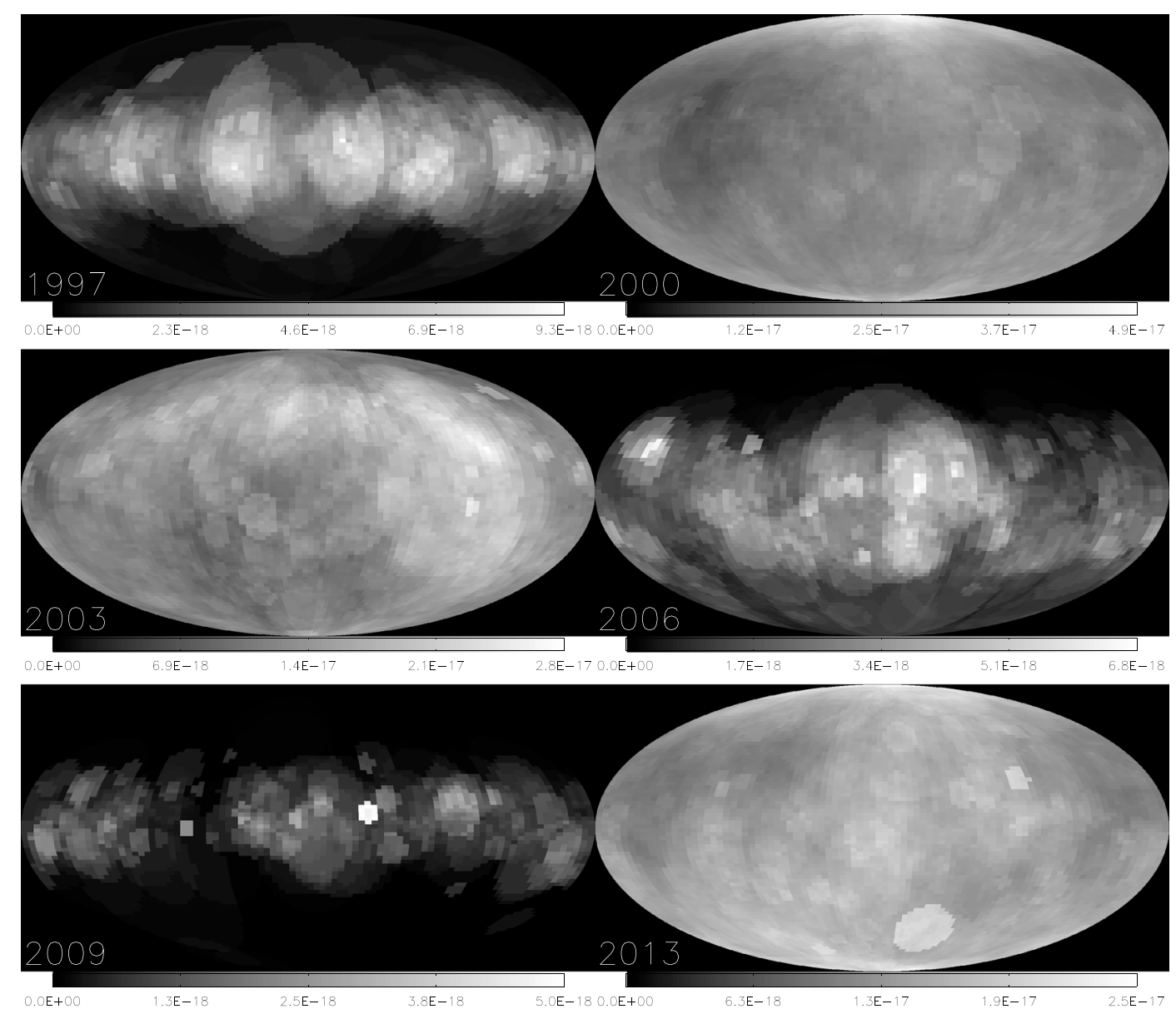

Figure 4. Synoptic maps of the heliospheric proton mass flux of CMEs at 1 AU for 6 years using the Mollweide projection. The grey scales have units of $\mathrm{g} \mathrm{cm}^{-2} \mathrm{~s}^{-1}$, and their range is different for each panel.

1. CMEs listed in the ARTEMIS-II catalog but whose mass cannot be measured are neglected. It is however possible to estimate this "missing" mass by using the intensity parameter reported for all CMEs in the catalog, that is their total radiance measured on synoptic maps at $3 R_{\odot}$. As explained by Floyd et al. [2013], this intensity cannot be converted to mass but is related to it. This relationship can be inferred from those CMEs whose mass is measured. Further assuming that both groups with and without listed mass have globally the same kinematic properties, the estimated missing mass amounts to $\approx 16 \%$ of the measured mass.

2. CMEs may be elongated flux ropes rather than spherical caps. The orientation of these flux ropes are random so that their apparent width gives in average a good estimate of the cone angle of the assumed axisymmetric spherical cap model. In addition, flux ropes have been observed to rotate with little projection effects supporting the prevalent view of constant angular width for CMEs [Vourlidas et al., 2011].

3. The mass of each CME is assumed to be uniformly distributed over its spherical cap representation. To assess the consequence of this idealized configuration, we have tested different mass distributions over the cap and concluded that it has no impact on the mass flux maps owing to their low spatial resolution.

4. Considering that $98.5 \%$ of CMEs are close to the plane of the sky at the time of the LASCO observations should not introduce any significant distortion in their longitudinal distribution since we are accumulating a large number of events over a year. In addition, the SWAN solar wind data to be used for comparison lack longitudinal information.

5. The detection of the CMEs is performed at $3 R_{\odot}$, but the flux calculations are performed at $1 \mathrm{AU}$. Propagation times are not important owing to the low spatial resolution of the yearly maps.

The most serious limitation results from the interpretation of the central position angle of a CME as it is complicated by the "projected" nature of the features seen in coronagraph images. A radial structure at true 


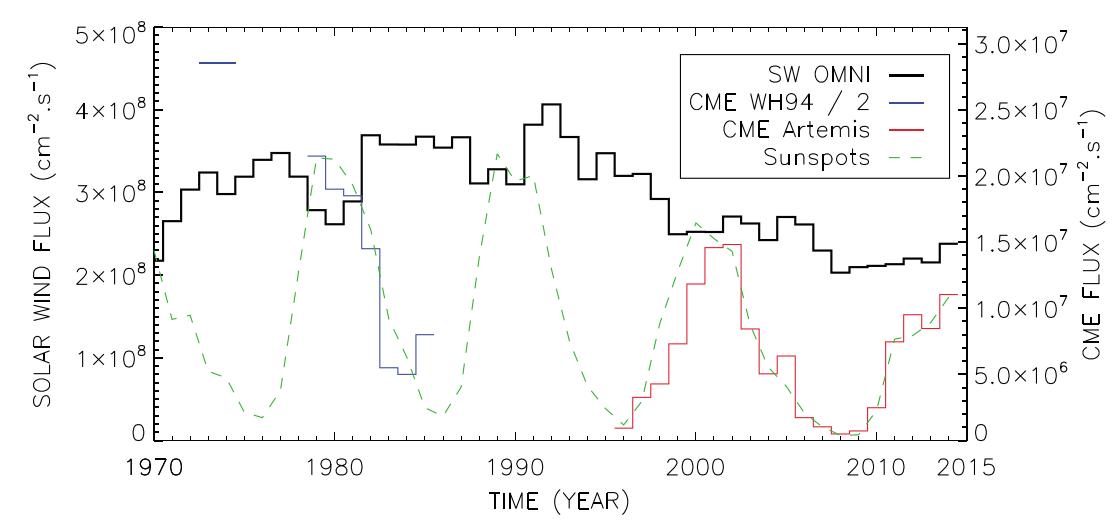

Figure 5. Temporal variation of the CMEs and solar wind proton fluxes at $1 \mathrm{AU}$ in the near-ecliptic region. The results of Webb and Howard [1994] covering the years 1973 and 1978-1985 are divided by a factor 2 to limit the scale. The (unscaled) sunspot number is overplotted to illustrate solar activity.

heliographic latitude $\lambda$ will be seen at the apparent latitude $\Lambda=\lambda$ if it lies in or close to the plane of the sky, but otherwise at an apparent latitude $\Lambda>\lambda$, the difference increasing with increasing distance from this plane. The usual convention is to neglect those effects and to simply assume that $\lambda=\Lambda$. Hundhausen [1993] investigated the consequences of this approximation and concluded that structures seen at apparent latitudes greater than $\approx 60^{\circ}$ could have true latitudes anywhere in the range 45 to $90^{\circ}$.

\subsection{Comparison of the CME and Solar Wind Mass Fluxes in the Ecliptic}

Past comparisons of these fluxes (as summarized in section 1) have been performed in the near-ecliptic region using in situ spacecraft data, and we therefore follow this standard practice. The CME mass flux as calculated in the above section is averaged in a $10^{\circ}$ wide equatorial band so that we can neglect the effect of the inclination of the solar axis. The solar wind mass flux data come from the OMNI database (http://omniweb.gsfc.nasa.gov/) which aggregates in situ measurements of the solar wind performed by many spacecraft starting in 1963 . The annualized values of both fluxes are displayed in Figure 5 and the corresponding ratio in Figure 6. Past results from Hildner [1977], Howard et al. [1985], Webb and Howard [1994], and Jackson and Howard [1993] presented in section 1 are overplotted as well as the (unscaled) sunspot number to reflect the solar activity. It is readily seen that the CME mass flux in the near-ecliptic band tracks very well the solar cycle, unlike the solar wind mass flux which remained roughly constant over solar cycles 21 and 22 and underwent a steady decrease at the onset of cycle 23 to reach a constant low level since the minimum of solar cycle $23 / 24$. Since the mass flux is the product of density times velocity, the flow velocity of the solar wind is important and likely one reason that the SW mass flux is relatively constant over the solar cycle as already noted by Webb and Howard [1994]. The slow wind and solar activity, e.g., density, is more important near maximum, whereas high-speed

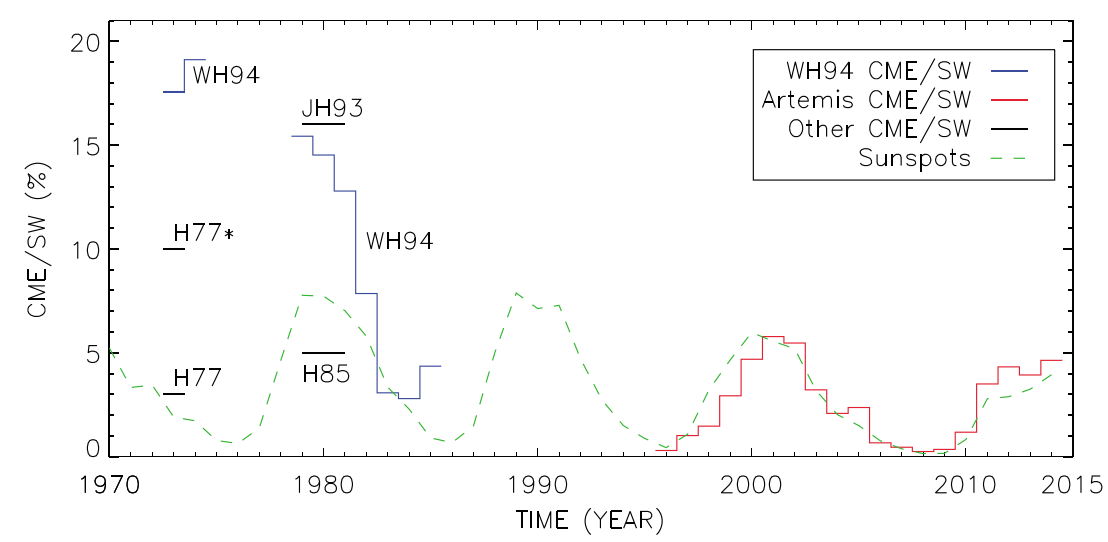

Figure 6. Temporal variation of the ratio of the CME proton flux to that of the solar wind. Past results are from H77 - Hildner [1977], H77* — later correction by Webb and Howard [1994], H85 — Howard et al. [1985], JH93 — Jackson and Howard [1993], and WH94-Webb and Howard [1994]. The (unscaled) sunspot number is overplotted to illustrate solar activity. 
streams, e.g., velocity, dominate during the declining and minimum phases. The fact that the CME/SW ratio tracks the solar cycle, a trend already noticed by Webb and Howard [1994] therefore entirely results from the temporal behavior of the CME mass flux. Our results show that this ratio varied from at most $0.5 \%$ during the solar minima of cycles $22 / 23$ and $23 / 24$ to $6 \%$ and $5 \%$ at the maximum of solar cycles 23 and 24 , respectively.

The past results of Hildner [1977] and Howard et al. [1985] are in line with ours, whereas those of Jackson and Howard [1993] and Webb and Howard [1994] (including their revised value of Hildner [1977]) indicate larger ratios, up to $16 \%$ for the maximum of solar cycle 21 and even $17.5 \%$ during the last stage of the declining phase of cycle 20. LASCO is far more sensitive and better calibrated than previously flown coronagraphs, and the procedure of estimating the mass of CMEs has been validated by independent determinations; our results should therefore be more accurate than those previously published. Past estimations required extensive corrections and revisions that may partly explained the differences with our results. For instance, Webb and Howard [1994] had to use published average CME and SW mass values from different sources for Skylab, SMM, Solwind, and the SW data, and they had to intercalibrate ecliptic versus global estimates of CME mass and the detection efficiency of the early coronagraph data. We estimate that our mass determination is uncertain by a typical factor of 2 to 3, most sources of error being nonsystematic, thus averaging out when considering a large number of CMEs. The most obvious systematic error results from the assumption that the bulk of the CMEs lie in the plane of the sky, thus resulting in a systematic underestimation of their mass. However, the comparison of our masses with those of the SECCHI CMEs which are better constrained does not indicate any dramatic underestimation for most of them.

Finally, it may be argued that solar cycle 21 was stronger than cyles 23 and 24 resulting in a higher occurrence rate of CMEs. As a simple exercise, let us scale the CME flux by the ratio of the maximum values of the sunspot number of solar cycles 21 and 23 that is a factor $\approx 1.3$. Then our ratio of $6 \%$ would be raised to $7.8 \%$ but which must be further corrected by the difference in the solar wind flux, ending up in an extrapolated (from our result) ratio of $\approx 7 \%$ for the maximum of cycle 21 to be compared to the $16 \%$ obtained by Webb and Howard [1994]. Based on present knowledge, it is difficult to conclude whether this reflects a true physical effect of solar activity on the occurrence rate of CMEs (which would therefore not be correctly described by the sunspot number) or observational and/or processing biases.

\section{Solar Wind Mass Flux}

\subsection{SWAN Solar Wind Data Set}

The SOHO/SWAN instrument is composed of two identical units placed on opposite sides of the SOHO spacecraft [Bertaux et al., 1995]. Each unit is a Lyman $\alpha$ photometer equipped with a periscopic scanning system that allows for a full coverage of half the sky in $24 \mathrm{~h}$. Data from both units are combined to produce full-sky images of the interplanetary glow at Lyman $\alpha$ as seen from the position of SOHO. SWAN has been in continuous operation since early 1996. At the beginning of the SOHO mission, SWAN performed an average of four full-sky observations per week. Since 2007, the full-sky observations have been performed on a daily schedule.

The interplanetary glow is caused by the backscatter of the $\mathrm{H}$ Lyman $\alpha$ photons emitted by the Sun by hydrogen atoms from the local interstellar medium flowing inside the heliosphere. These atoms enter the heliosphere with a bulk velocity close to $26 \mathrm{~km} / \mathrm{s}$ due to the relative motion of the solar system in the local interstellar cloud. Many of these atoms reach the inner heliosphere and the vicinity of the Sun before being ionized by photoionization, and prominently, by charge exchange with solar wind solar protons. Let $\beta_{\mathrm{ex}}$ and $\beta_{\mathrm{EUV}}$ denote the charge exchange ionization rate and the photoionization rate respectively and $\beta_{\text {tot }}$ their sum. Both ionization rates vary with the inverse of the square of the distance to the Sun and so does their sum. Therefore, using this radial dependence, maps of the Lyman $\alpha$ backscattered intensity in the interplanetary medium allow deriving the hydrogen ionization rate at $1 \mathrm{AU}$.

Quémerais et al. [2006] summarized the inversion procedure implemented to obtain the distribution of total hydrogen ionization rate from the SWAN maps. They used a nonlinear least squares fit to the data based on an improved version of the hot model developed by Lallement et al. [1985] with corrections for an anisotropic ionization rate varying with heliographic latitude and any values for the radiation pressure coefficient. The radiation pressure acting on hydrogen atoms is defined by the solar flux at the time of observation. Because the solar wind rotation is fast compared to the timescale of the considered ionization process in the interplanetary medium, there is no longitudinal information for the $\mathrm{H}$ ionization rate. On the other hand, variations of 

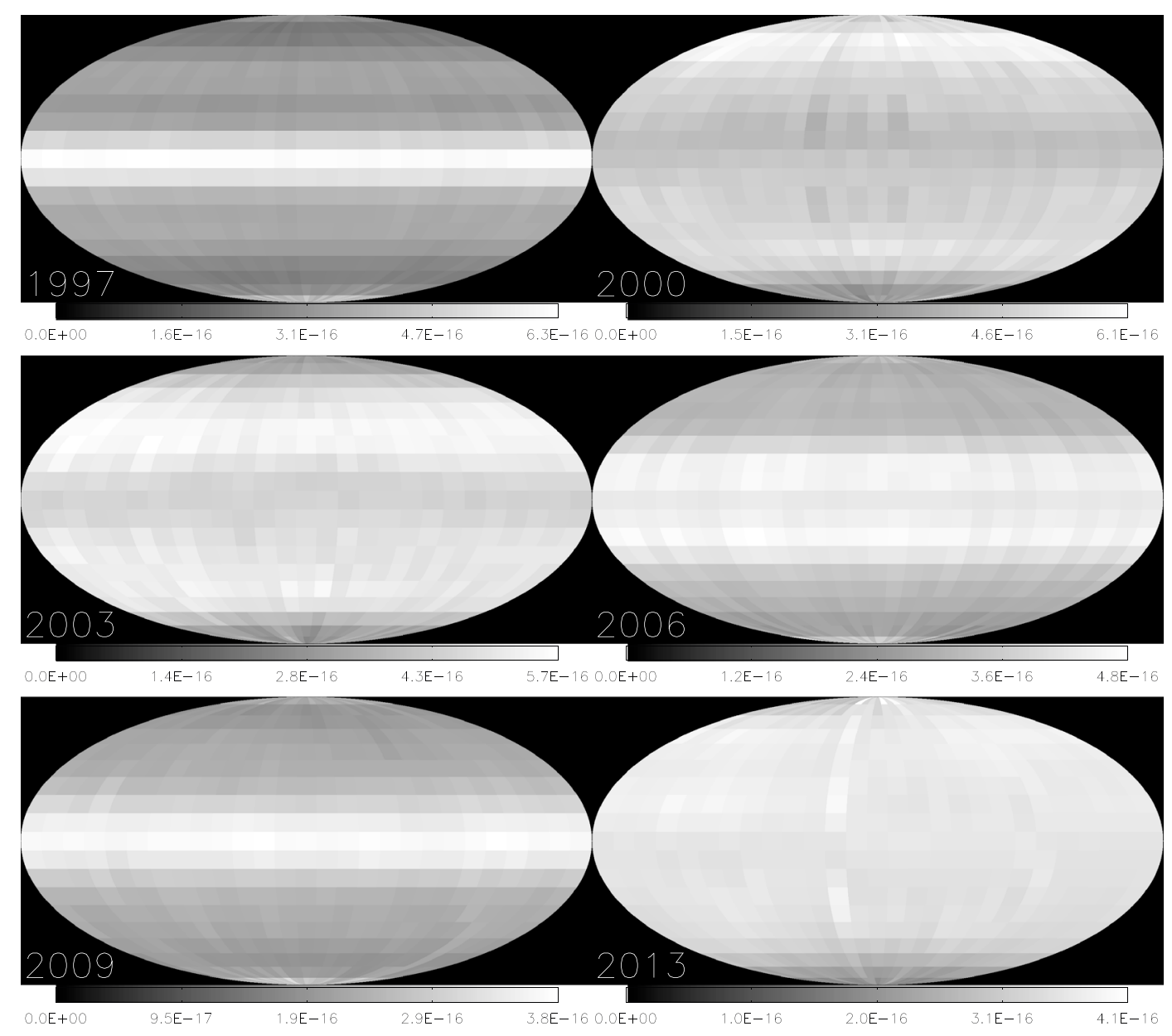

Figure 7. Synoptic maps of the proton mass flux of the solar wind from SWAN for 6 years using the Mollweide projection. The grey scales have units of $\mathrm{g} \mathrm{cm}^{-2} \mathrm{~s}^{-1}$, and their range is different for each panel.

the $\mathrm{H}$ ionization rate with the heliographic latitude are imprinted on the $\mathrm{H}$ interplanetary glow and can be recovered during the inversion of the SWAN full-sky maps.

The inversion scheme is applied to each full-sky map obtained by SWAN. The input parameters are the interstellar wind parameters, the radiation pressure coefficient at the time of observation, the solar illuminating flux, and the geometry of the observations. The output consists of the values of the $\mathrm{H}$ ionization rate at $1 \mathrm{AU}$ and the corresponding uncertainties averaged over 19 bins of heliographic latitude from the south to the north solar poles. As can be expected, uncertainties affecting the values derived over the poles are significantly larger than those obtained in the equatorial region. After inversion, a running average over a solar rotation is applied to mitigate this effect.

Equation (1) below shows how the solar wind mass flux at $1 \mathrm{AU}$ is retrieved from the $\mathrm{H}$ ionization rate at $1 \mathrm{AU}$. This equation expresses the relationship between the solar wind mass flux and the total ionization rate of hydrogen atoms at $1 \mathrm{AU}$ :

$$
\beta_{\mathrm{tot}}=\beta_{\mathrm{EUV}}+\beta_{\mathrm{ex}}=\beta_{\mathrm{EUV}}+\sigma\left(V_{s} w\right) \cdot \phi_{\mathrm{sw}} .
$$

To derive the solar wind mass flux $\phi_{\mathrm{Sw}}$, we first estimate the value of the photoionization rate $\beta_{\mathrm{EUV}}$ at the time of observation from solar measurements. Then, we subtract this estimate of $\beta_{\mathrm{EUV}}$ from the total ionization rate.

The charge exchange ionization rate $\beta_{\mathrm{ex}}$ is the product of the solar wind mass flux $\phi_{\mathrm{sw}}$ with the charge exchange cross section between the $\mathrm{H}$ atoms and solar wind protons. This cross-section $\sigma\left(V_{s} w\right)$ slightly depends upon the solar wind velocity. Therefore, the SWAN maps allow us to derive the total ionization rate and after correcting for the solar photoionization rate, the charge exchange ionization rate at all 

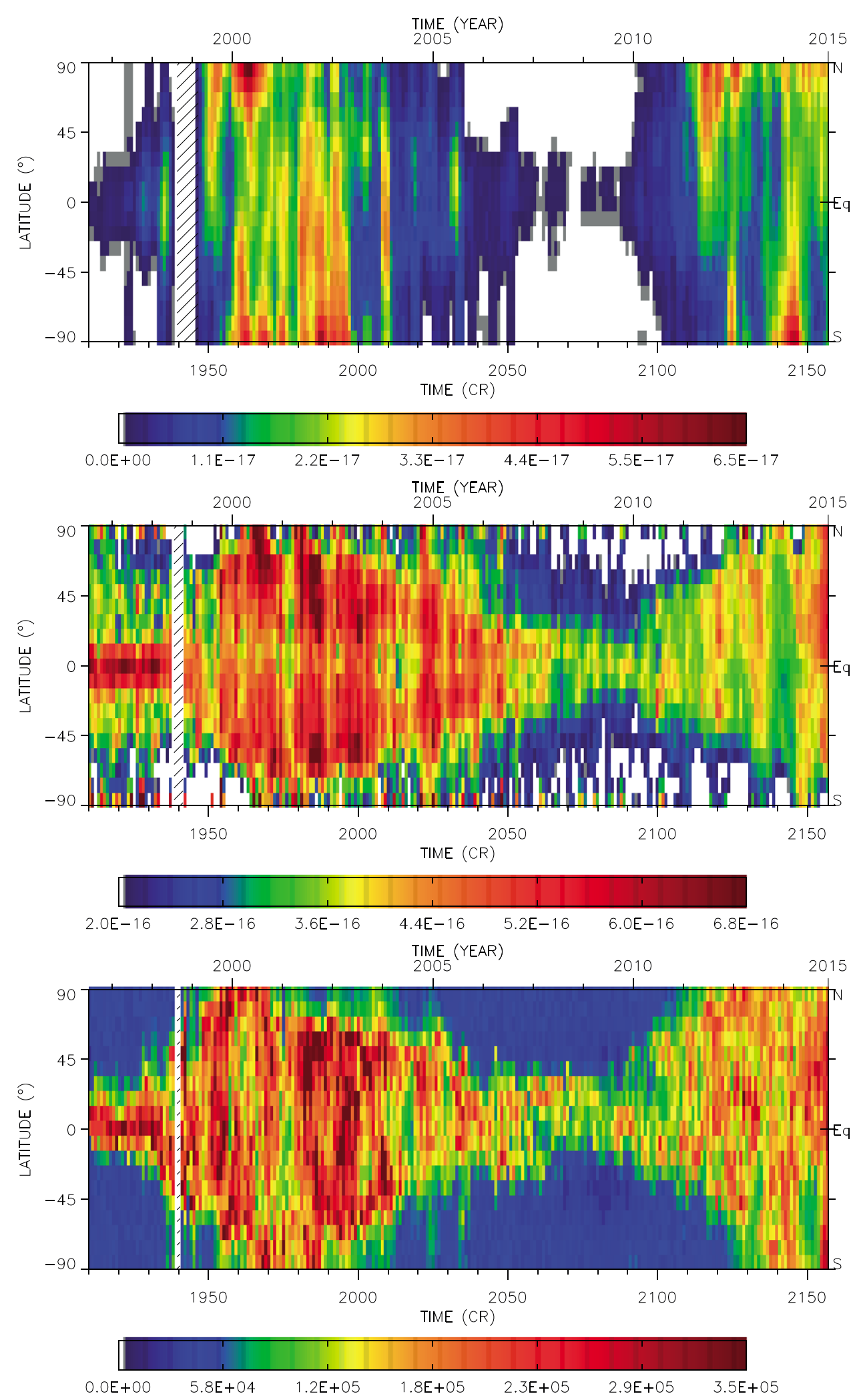

Figure 8. Heliolatitudinal distributions of the proton mass flux of the (top) CMEs, of the (middle) solar wind, and of the (bottom) coronal electron density at a heliocentric distance of $3.5 \mathrm{R}_{\odot}$. The hatched columns correspond to missing data (when SOHO lost its pointing). The upper two color scales have units of $\mathrm{g} \mathrm{cm}^{-2} \mathrm{~s}^{-1}$. The lower color scale has units of $\mathrm{cm}^{-3}$. 


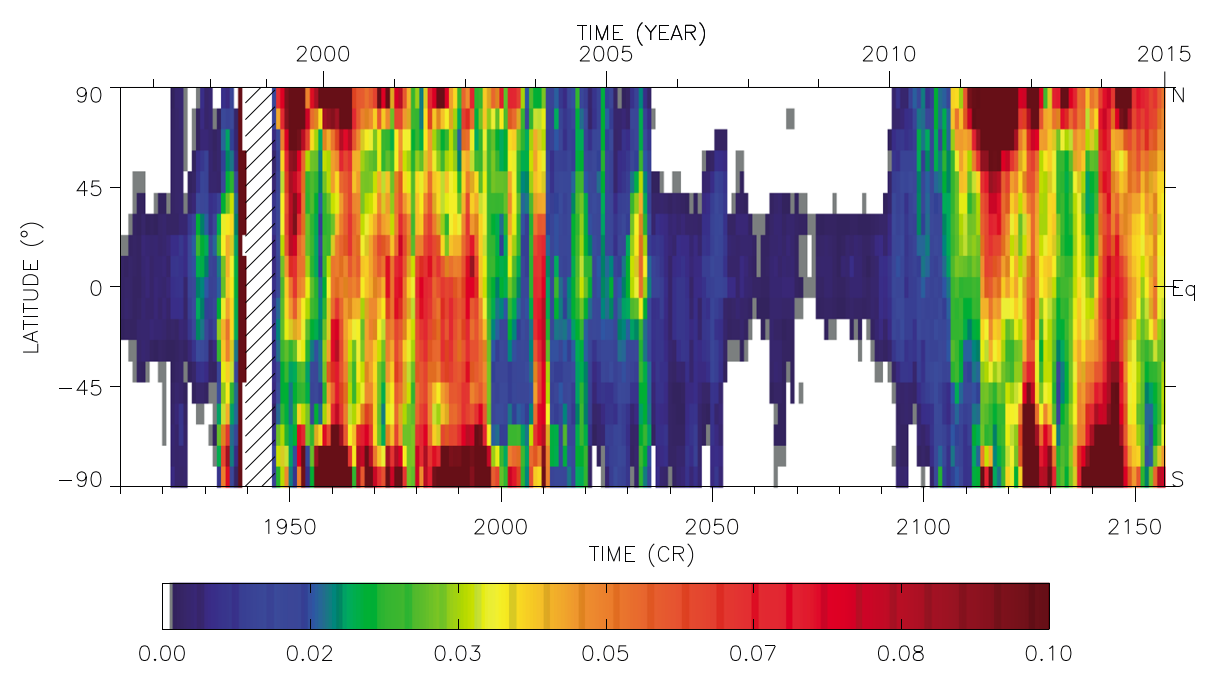

Figure 9. Heliolatitudinal distribution of the ratio of the CME proton mass flux to that of the solar wind. The hatched column corresponds to missing data (when SOHO lost its pointing).

heliographic latitudes. In the ecliptic plane, this rate can be computed from solar wind measurements as given by the OMNI database. Therefore, the rates derived from the SWAN maps can be compared to the values derived from the solar wind measurements in the ecliptic plane. Outside of the ecliptic plane, only SWAN provides measurements on a regular basis.

The ionization rate values derived from SWAN are linearly dependent upon the solar illuminating flux, the SWAN absolute calibration, and the hydrogen number density at large distances from the Sun assumed as an input to the model. Therefore, given that at least the last two of these three parameters are not accurately known and are still a matter for debate, we have scaled the results of the inversion in the ecliptic plane to the values computed from the OMNI database and averaged over one solar rotation. This ensures that the results of our inversion is in agreement with the in situ solar wind measurements. The result is shown in Figure 11 of Quémerais et al. [2006].

The solar wind mass flux is obtained by dividing the ionization rate $\beta_{\text {ex }}$ at all latitudes by the charge exchange cross section using the mean velocity of the solar wind derived in the ecliptic plane from the OMNI database. This adds an additional uncertainty as the solar wind velocity varies with heliographic latitude. However, it is found that the charge exchange cross-section dependence on velocity is small compared to the uncertainties introduced by the inversion of the SWAN data at high heliographic latitudes. Therefore, we have chosen to neglect this effect in our inversion.

\subsection{Calculation of the Solar Wind Mass Flux}

The SWAN solar wind data from 1996 to 2014 inclusive were converted to synoptic maps in order to be directly compared with those of the CMEs. They are equivalent to a set of daily columns of 19 pixels, and synoptic maps composed of 27 columns and 19 rows (each row corresponding to a latitude band) are generated for each Carrington rotation by resampling these data to their nearest neighbor. In case of missing columns, they are set to the average value over the rotation for each latitude band. Annual averages of the synoptic maps are also generated, with the data from the first and last Carrington rotations used in full but weighted proportionally to how much they overlap with the civil year. Six of these annual maps are displayed in Figure 7. As expected, the solar wind mass flux exhibits a higher concentration in the equator region during the periods of low solar activity and a more isotropic distribution during the periods of high activity.

\subsection{Comparison of the CME and Solar Wind Mass Fluxes in the Heliosphere}

Figure 8 displays the heliolatitudinal distributions of the mass flux of the CMEs and of the solar wind derived from SWAN data. They are obtained by averaging the images of the mass flux for each Carrington rotation over the longitude and stacking them along the time axis. These maps have a dimension of $247 \times 19$ pixels, where each column therefore corresponds to a Carrington rotation and each row to the latitude band dictated by the low resolution of the SWAN data $\left(10^{\circ}\right)$. Globally, they both follow the standard pattern of many proxies of solar activity with concentration in the equatorial band during solar minima and widespread latitudinal 


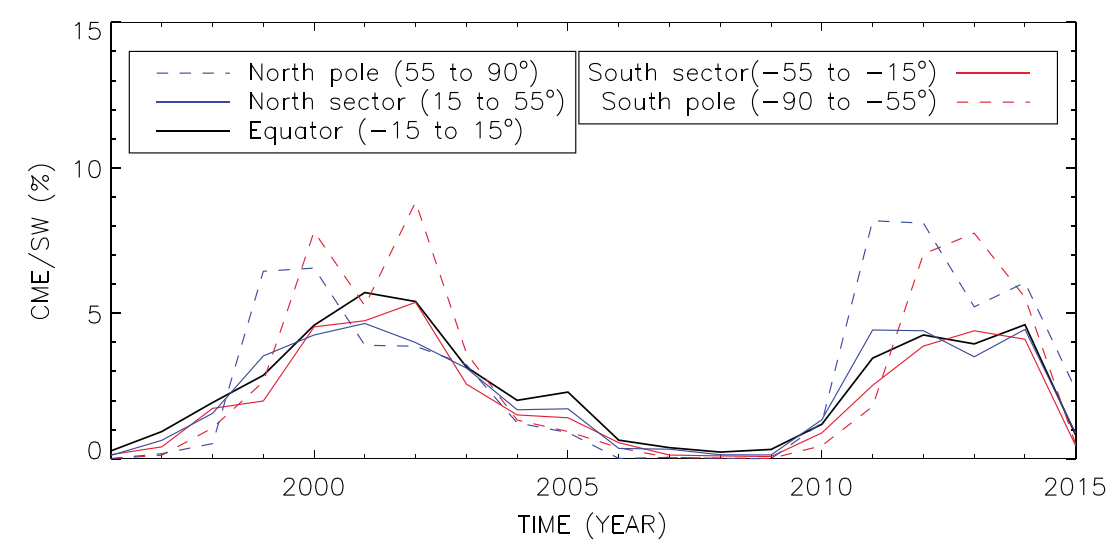

Figure 10. Temporal variation of the ratio of the CME proton mass flux to that of the solar wind in several latitude bands.

expansion during the maxima with an asymmetric pattern between the rising and declining phases (butterfly diagram). There are however subtle differences prominently during the maxima of the solar cycle: some local extrema (either minimum or maximum) may be present in the CME distribution and absent in that of the solar wind and vice versa. The most striking differences concern the maxima present only in the distribution of CMEs at high latitudes. As already discussed in section 3.2, they most likely result from the bias affecting the determination of latitude of those CMEs. Figure 8 further displays the heliolatitudinal distribution of the coronal electron density at a heliocentric distance of $3.5 \mathrm{R}_{\odot}$ computed by inversion of the LASCO $p B$ images as described by Lamy et al. [2014]. This map is however different from those presented by these authors as the east and west synoptic maps are presently combined altogether with proper orientation during the duration of each Carrington rotation. The similarity with the distribution of the solar wind flux is striking and can be observed in many high-frequency structures, in contrast with the case of CMEs. In a broad stroke, the electron density and solar wind fluxes only differ by the velocity of the solar wind which is distinctively larger in the coronal holes, and this is what we observe in the two maps. There is a delay of typically 6 months between the Lyman $\alpha$ emission measured by SWAN and the actual properties of the solar wind, but this is hardly noticeable in the maps.

Figure 9 displays the heliolatitudinal distribution of the ratio of the CMEs and solar wind mass fluxes which appears heavily controlled by that of the CMEs: note, in particular, the large values in the polar regions during the two maxima of solar activity. This is confirmed by Figure 10 where we display the above ratio averaged in broad latitudinal bands. The curve pertaining to the equatorial band is similar to that of the near-ecliptic region (Figure 6), and their common behavior holds as well to higher latitudes, typically up to $55^{\circ}$ north and south. In the two polar caps, and consistent with the above results, the ratio peaks at larger values, up to $\sim 10 \%$. As already mentioned, these values most likely result from the bias affecting the determination of the true latitude of "polar" CMEs.

\section{Conclusion}

We have reexamined the question of the contribution of coronal mass ejections to the solar wind mass flux in the light of 19 years (1996-2014) of observations performed by the LASCO and SWAN instruments both on board SOHO, supplemented by in situ data of the OMNI database. In a preliminary effort, we have shown that the CME masses reported in the ARTEMIS catalog of LASCO CMEs are in reasonable agreement with those based on combined observations with the twin STEREO/SECCHI coronagraphs. We found that the fractional contribution of CMEs to the solar wind mass flux closely tracks the solar cycle as already noticed on the basis of past Skylab and Solwind measurements but emphasized that this prominently results from the temporal variation of the production of CMEs. In the near-ecliptic region, the contribution of CMEs was negligibly small during the solar minima of cycles $22 / 23$ and $23 / 24$ and rose to, respectively, $6 \%$ and $5 \%$ at the maximum of solar cycles 23 and 24. These values differ from those reported in the past by some researchers, up to $16 \%$ at the maximum of solar cycle 21 , but we argued that this difference could not be explained by the different strengths of the solar maxima if the CME flux rate were to track the sunspot number. It is thus unclear whether this reflects a true physical effect of solar activity on the production of CMEs or observational/methodology biases. We extended our study to all latitudes and found that our results hold at latitudes up to $\sim 55^{\circ}$. 


\section{Acknowledgments}

We thank M. Temmer for providing the CME masses derived from the SECCHI observations and J. Wojak for preparing the figure displaying the heliolatitudinal distribution of the electron density. We acknowledge constructive remarks from the two reviewers who help us clarifying several aspects. The LASCO-C2 project at the Laboratoire d'Astrophysique de Marseille and the SWAN project at the Laboratoire Atmosphères, Milieux et Observations Spatiales are funded by the Centre National d'Etudes Spatiales (CNES). LASCO was built by a consortium of the Naval Research Laboratory, USA, the Laboratoire d'Astrophysique de Marseille (formerly Laboratoire d'Astronomie Spatiale), France, the Max-Planck-Institut für Sonnensystemforschung (formerly Max Planck Institute für Aeronomie), Germany, and the School of Physics and Astronomy, University of Birmingham, UK. SWAN was developed as a cooperation between the Laboratoire Atmosphères, Milieux et Observations Spatiales, France, and the Finnish Meteorological Institute, Finland. The primary data supporting the analysis are available at (http://lasco-www.nrl.navy.mil/) and (http://swan.projet.latmos.ipsl.fr/). The secondary data produced at the Laboratoire d'Astrophysique de Marseille and at the Laboratoire Atmosphères, Milieux et Observations Spatiales are available, respectively, from the first and third authors upon request and subject to an agreement. $\mathrm{SOHO}$ is a project of international cooperation between ESA and NASA.
At higher latitudes in the polar caps, we found larger ratios, up to $\sim 10 \%$ at solar maxima but suspect that this is likely biased by the inherent limitation of determining the true latitude of the involved CMEs. We finally conclude that during the last 19 years (1996-2014), CMEs contributed marginally to the solar wind mass flux and negligibly during the solar cycle minima.

\section{References}

Bein, B. M., M. Temmer, A. Vourlidas, A. M. Veronig, and D. Utz (2013), The height evolution of the "true" coronal mass ejection mass derived from STEREO COR1 and COR2 observations, Astrophys. J., 768(1), 12, doi:10.1088/0004-637X/768/1/31.

Boursier, Y., P. Lamy, and A. Llebaria (2009a), Three-dimensional kinematics of coronal mass ejections from STEREO/SECCHI-COR2 observations in 2007-2008, Sol. Phys., 256, 131-147, doi:10.1007/s11207-009-9358-1.

Boursier, Y., P. Lamy, A. Llebaria, F. Goudail, and S. Robelus (2009b), The ARTEMIS catalog of LASCO coronal mass ejections. Automatic recognition of transient events and marseille inventory from synoptic maps, Sol. Phys., 257(1), 125-147, doi:10.1007/s11207-009-9370-5. Brueckner, G. E., et al. (1995), The Large Angle Spectroscopic Coronagraph (LASCO), Sol. Phys., 162(1-2), 357-402, doi:10.1007/BF00733434. Bertaux, J. L., et al. (1995), SWAN: A study of Solar Wind Anisotropies on SOHO with Lyman-alpha sky mapping, Sol. Phys., 162(1-2), 403-439, doi:10.1007/BF00733435.

Colaninno, R. C., and A. Vourlidas (2009), First determination of the true mass of coronal mass ejections: A novel approach to using the two STEREO viewpoints, Astrophys. J., 698(1), 852-858, doi:10.1088/0004-637X/698/1/852.

Floyd, O., P. Lamy, Y. Boursier, and A. Llebaria (2013), ARTEMIS II: A second-generation catalog of LASCO coronal mass ejections including mass and kinetic energy, Sol. Phys., 288(1), 269-289, doi:10.1007/s11207-013-0281-0.

Hildner, E. (1977), Mass ejections from the corona into interplanetary space, in Studii of Travelling Interplanetary Phenomena, edited by M. A. Shea et al., 3 p., D. Reidel, Dordrecht, Netherlands.

Howard, R. A., N. R. Sheeley, M. J. Koomen Jr., and D. J. Michels (1985), Coronal mass ejections: 1979-1981, J. Geophys. Res., 90, 8173-8191, doi:10.1029/JA090iA09p08173.

Hundhausen, A. J. (1993), Sizes and locations of coronal mass ejections - SMM observations from 1980 and 1984-1989, J. Geophys. Res., 98, 177-200, doi:10.1029/93JA00157.

Jackson, B. V., and R. A. Howard (1993), A CME mass distribution derived from Solwind coronagraph observations, Sol. Phys., 148, $359-370$. Lamy, P., T. Barlyaeva, A. Llebaria, and O. Floyd (2014), Comparing the solar minima of cycles 22/23 and 23/24: The view from LASCO white light coronal images, J. Geophys. Res. Space Physics, 119(1), 47-58, doi:10.1002/2013JA019468.

Lallement, R., J. L. Bertaux, and V. G. Kurt (1985), Solar wind decrease at high heliographic latitudes detected from Prognoz interplanetary Lyman alpha mapping, J. Geophys. Res., 90(1), 1413-1423, doi:10.1029/JA090iA02p01413.

Quémerais, E., R. Lallement, S. Ferron, D. Koutroumpa, J.-L. Bertaux, E. Kyrölä, and W. Schmidt (2006), Interplanetary hydrogen absolute ionization rates: Retrieving the solar wind mass flux latitude and cycle dependence with SWAN/SOHO maps, J. Geophys. Res., 111, A09114, doi:10.1029/2006JA011711.

Vourlidas, A., R. C. Colaninno, T. Nieves-Chinchilla, and G. Stenborg (2011), The first observation of a rapidly rotating coronal mass ejection in the middle corona, Astrophys. J. Let., 733, L23 - L28, doi:10.1088/2041-8205/733/2/L23.

Webb, D. F., and R. A. Howard (1994), The solar cycle variation of coronal mass ejections and the solar wind mass flux, J. Geophys. Res., 99 , 4201-4220, doi:10.1029/93JA02742. 